\title{
Um caso exuberante de eritema nodoso associado a desogestrel
}

Filipa Sofia Silva, ${ }^{1}$ Pedro Carreira, ${ }^{2}$ Sónia Serra ${ }^{3}$

\section{RESUMO}

Introdução: O eritema nodoso consiste numa erupção cutânea eritematosa aguda, do tipo nodular, que habitualmente se encontra limitada à face anterior das pernas. As principais causas são as infeções estreptocócicas nas crianças e as infeções estreptocócicas e a sarcoidose nos adultos.

Descrição do caso: Os autores apresentam o caso de uma mulher de 33 anos, encaminhada a partir do serviço de urgência por poliartralgias com uma semana de evolução, às quais se associou o aparecimento de nódulos eritematosos dolorosos nos membros inferiores, sugestivos de eritema nodoso. Descreve-se todo o estudo e acompanhamento realizado em consulta de medicina interna, com identificação da etiologia, a toma de desogestrel com exclusão de outras etiologias possíveis.

Comentário: O quadro clínico teve uma evolução favorável, com resolução completa dos sintomas e das lesões cutâneas, após esclarecimento etiológico e abordagem terapêutica em conformidade.

Palavras-chave: Eritema nodoso; Etiologia; Anticoncecionais.

\section{INTRODUÇÃO}

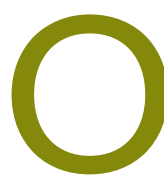

eritema nodoso consiste numa erupção cutânea eritematosa aguda, do tipo nodular, que habitualmente se encontra limitada à face anterior das pernas. ${ }^{1-2}$

$\mathrm{O}$ pico de incidência do eritema nodoso ocorre entre os 18 e os 34 anos de idade, sendo as mulheres mais afetadas que os homens, com uma relação de quatro para um. ${ }^{1,3}$

As principais causas de eritema nodoso nas crianças são as infeções estreptocócicas. ${ }^{1}$ Nos adultos as principais causas são as infeções estreptocócicas e a sarcoidose, correspondendo a cerca de 10 a $22 \%$ do número total de casos. ${ }^{1,3-4}$ Existem outros agentes causadores de eritema nodoso, como a Yersinia enterocolitica, o $M y$ coplasma pneumoniae, a Salmonella, o Campylobacter e o Mycobacterium tuberculosis. ${ }^{1,4-6}$ Alguns fármacos foram já identificados como causa de eritema nodoso e

1. Médica Interna de Medicina Interna. Centro Hospitalar de Setúbal, EPE. Setúbal, Portugal.

2. Médico Assistente Hospitalar de Medicina Interna. Centro Hospitalar de Setúbal, EPE. Setúbal, Portugal.

3. Médico Assistente Hospitalar Graduada de Medicina Interna. Centro Hospitalar de Setúbal, EPE. Setúbal, Portugal. incluem as sulfonamidas, sulfonilureias e, mais recentemente, os contracetivos orais (principalmente relacionados com a toma de estrogénios), sendo apontados como agentes etiológicos num número crescente de casos. ${ }^{1,3-5} \mathrm{O}$ eritema nodoso pode manifestar-se como parte integrante do quadro das doenças inflamatórias intestinais e pode surgir também no contexto de doenças hematológicas, doenças autoimunes e situações muito particulares, como a gravidez, principalmente no segundo trimestre. ${ }^{1,3,7}$

$\mathrm{Na}$ sua forma mais comum, a doença manifesta-se com aparecimento de febre e artralgias, que ocorrem em mais de $50 \%$ dos doentes e podem anteceder o aparecimento dos nódulos cutâneos em duas a quatro semanas ou surgirem durante o aparecimento dos nódulos cutâneos. ${ }^{1,6}$ Os nódulos são tipicamente eritematosos, dolorosos, de bordos mal definidos e o seu diâmetro varia entre dois e seis centímetros. ${ }^{1,3,6}$ As lesões podem perdurar durante duas semanas ou continuar a aparecer durante três a seis semanas. ${ }^{1} \mathrm{O}$ curso da doença é habitualmente autolimitado, devendo o tratamento incluir o controlo sintomático e a restrição de atividade física na fase ativa da doença. ${ }^{1}$ 
A adequada articulação com as outras especialidades médicas, incluindo cuidados de saúde primários e restantes especialidades intra-hospitalares, é parte integrante da atividade clínica do médico de medicina interna e é uma mais-valia no tratamento e seguimento do utente. Este caso clínico pretende apresentar uma etiologia rara de eritema nodoso para a qual se deve estar alerta e, por outro lado, realçar a importância da adequada articulação entre as diferentes especialidades médicas, incluindo a medicina interna e a medicina geral e familiar. No decurso do caso clínico apresentado, a avaliação, seguimento e terapêutica realizada foram partilhadas entre o médico de medicina interna e o médico de medicina geral e familiar da utente, o que permitiu prestar os melhores cuidados médicos e manter a adequada continuidade dos cuidados prestados.

\section{DESCRIÇÃO DO CASO}

Os autores apresentam o caso de uma mulher de 33 anos, saudável, mãe de uma criança de quatro meses, gravidez e parto eutócico sem intercorrências, atualmente a amamentar e medicada desde o parto com desogestrel (um contracetivo progestativo), não se encontrando a realizar qualquer outra terapêutica. Previamente ao parto, apenas tinha utilizado o preservativo como método contracetivo.

Encaminhada a partir do serviço de urgência para a consulta de medicina interna por poliartralgia com uma semana de evolução, com início na articulação do joelho e tornozelo esquerdos com progressão disto proximal, à qual se associou aparecimento de nódulos eritematosos dolorosos na face anterior da perna esquerda e face anterior do joelho direito com um dia de evolução. Desde a observação no serviço de urgência até ser observada em consulta de medicina interna decorreu cerca de uma semana e meia e a doente não realizou qualquer terapêutica.

Na primeira consulta de medicina interna descreve um quadro compatível com poliartralgias generalizadas com grave incapacidade funcional, referindo também agravamento dos nódulos eritematosos (Figura 1). As manifestações cutâneas observadas são características de eritema nodoso e, no contexto do estudo etiológico, a utente foi submetida a análises séricas, exame sumário de urina (ESU) e radiografia de tórax. O ESU foi sugestivo de infeção do trato urinário (ITU), pelo que se

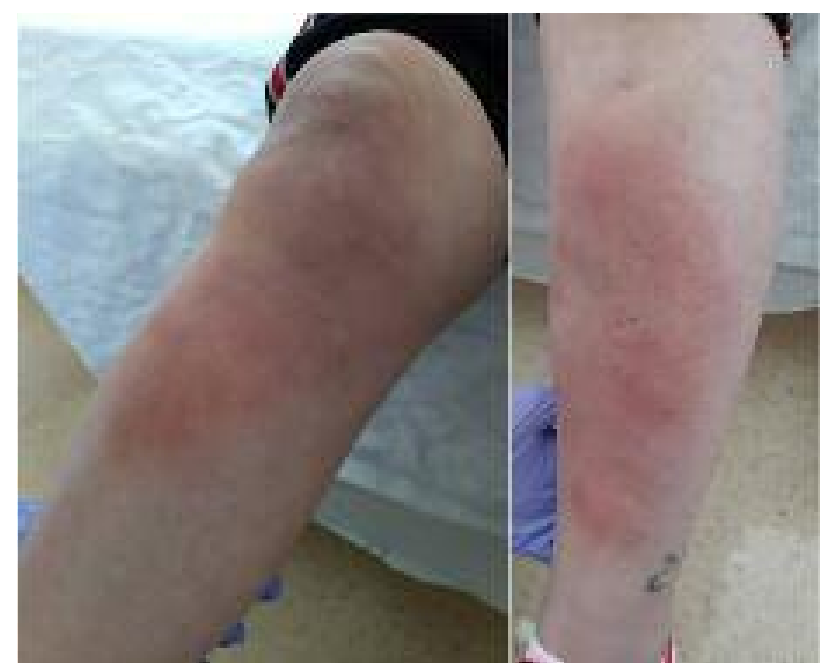

Figura 1. Lesões de eritema nodoso observadas na primeira consulta de medicina interna. Sob terapêutica apenas com desogestrel.

assumiu ITU assintomática, mas optou-se por iniciar antibioterapia empírica com amoxicilina ácido clavulânico após colheita de urocultura, dada a remota hipótese de se tratar de eritema nodoso com causa infeciosa subjacente. As restantes análises ficaram em curso. O contracetivo oral foi suspenso. A doente iniciou terapêutica com prednisolona $5 \mathrm{mg}$ por dia e paracetamol 1000mg para alívio sintomático, dois a três comprimidos por dia. A informação clínica da consulta de medicina interna foi partilhada com o médico de medicina geral e familiar da utente e, em consulta com o seu médico de família, a utente optou por manter o método contracetivo que utilizava previamente à gravidez - o preservativo.

Dos exames complementares de diagnóstico solicitados salienta-se: enzima de conversão da angiotensina negativo, função tiroideia sem alterações, beta $2 \mathrm{mi}-$ croglobulina normal, aumento ligeiro do adenosina desaminase (29U/L) com Igra negativo, eletroforese de proteínas com padrão inflamatório, radiografia de tórax sem alterações, hemocultura estéril, monoteste e antigenúria para Streptococcus negativos, serologia compatível com vacinação para hepatite B, com serologias negativas para HCV e HIV 1 e 2. Anticorpo para Mycoplasma, EBV-CA IgM, HSV1/2 IgM negativos. A reação de Huddleson e Rosa Bengala foram negativas. A pesquisa de anticorpo antinuclear e anticorpo anticitoplasma neutrófilo foram negativas (com anticorpo 


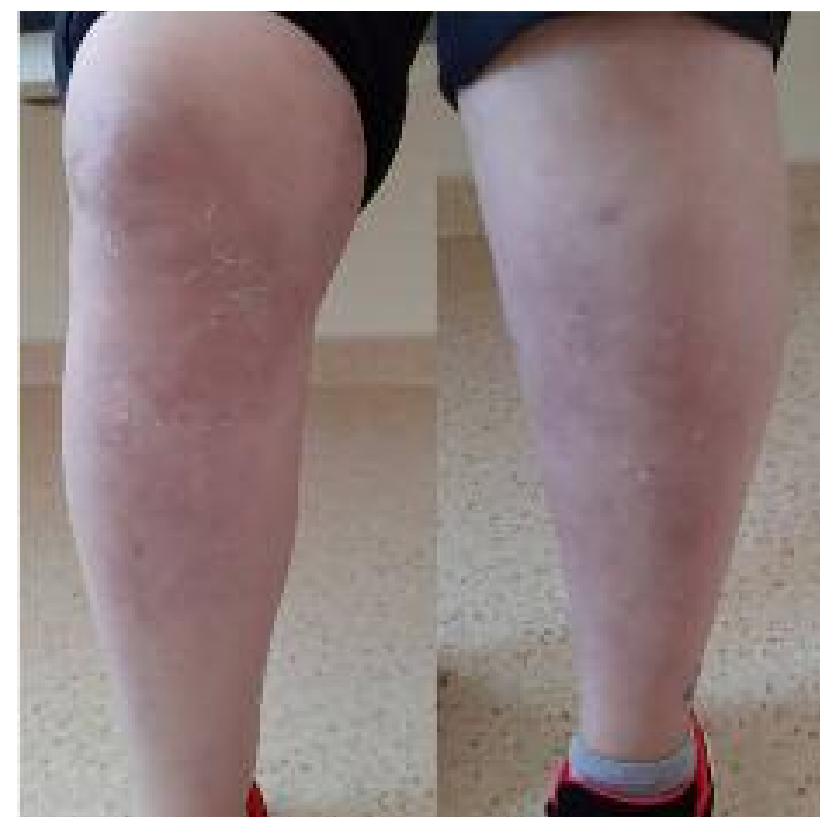

Figura 2. Lesões de eritema nodoso 15 dias após ter sido suspenso o contracetivo oral e iniciada prednisolona $5 \mathrm{mg}$ por dia.

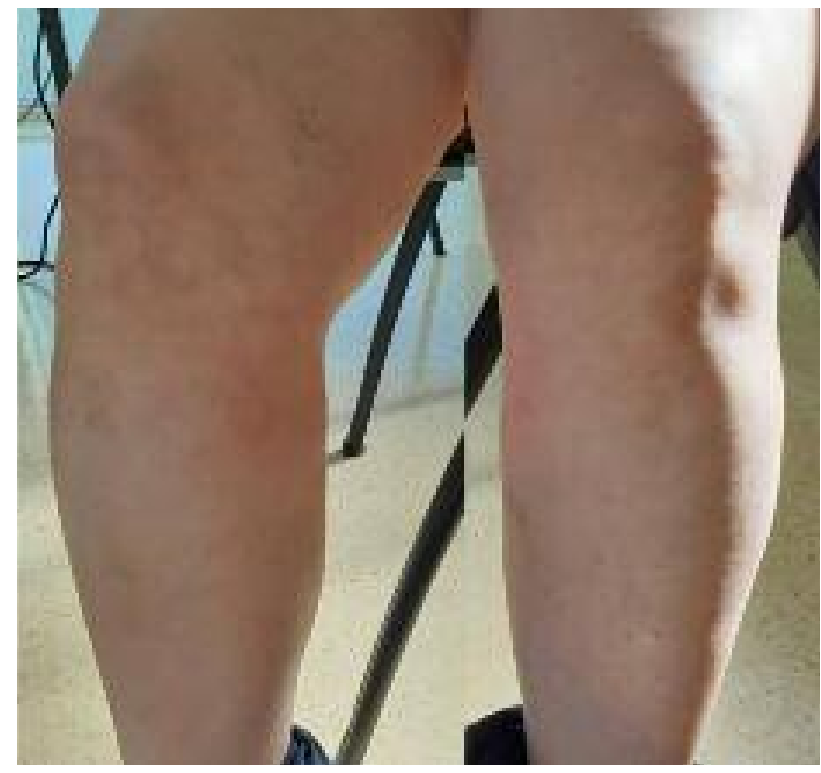

Figura 3. Ausência de lesões cutâneas ao vigésimo nono dia após suspensão de desogestrel e início de prednisolona $5 \mathrm{mg}$ por dia.

antiproteinase 3 menor que $0,2 \mathrm{UI} / \mathrm{mL}$ e anticorpo antimieloperoxidase menor que $0,2 \mathrm{UI} / \mathrm{mL}$ ).

Na segunda consulta de medicina interna, no oitavo dia após suspensão de desogestrel e oitavo dia de ad- ministração de prednisolona $5 \mathrm{mg} / \mathrm{d}$, apresenta melhoria das queixas álgicas e franca melhoria das lesões cutâneas. O resultado da urocultura realizada na primeira consulta isolou um Proteus mirabilis resistente a amoxicilina ácido clavulânico e sensível a cefalosporinas, pelo que iniciou antibioterapia dirigida de acordo com o resultado da urocultura, tendo cumprido terapêutica com cefuroxime 500mg, um comprimido de 12 em 12 horas. Manteve a toma de prednisolona $5 \mathrm{mg}$ por dia e o contracetivo oral suspenso.

Na terceira consulta de medicina interna, ao décimo quinto dia de prednisolona $5 \mathrm{mg} /$ dia e 15 dias após ter sido suspenso o desogestrel, mantém melhoria das artralgias e das lesões cutâneas (Figura 2), agora com pele mais descamativa.

Cerca de duas semanas depois (isto é, ao vigésimo nono dia após suspensão de desogestrel e início de prednisolona $5 \mathrm{mg} / \mathrm{d}$ ), quando reavaliada em consulta de medicina interna, a doente encontra-se assintomática e sem lesões cutâneas (Figura 3), pelo que suspende a terapêutica com prednisolona $5 \mathrm{mg} /$ dia e mantém indicação para não voltar a reintroduzir o desogestrel.

A utente foi reavaliada em consulta de medicina interna cerca de sete a nove meses depois da primeira avaliação em consulta, mantendo-se assintomática e com completa resolução das lesões cutâneas, sendo que o desogestrel não voltou a ser reintroduzido. Concomitantemente, a utente manteve acompanhamento pelo seu médico de família.

\section{COMENTÁRIO}

No decorrer da avaliação de um doente, quer seja em consulta ou quer em internamento, é necessário compreender o doente, a fisiopatologia da doença e conseguir isolar os fatores etiológicos para que o estudo e atitude terapêutica sejam inteiramente dirigidos.

Neste caso clínico, no curso do estudo etiológico, a utente foi submetida a análises séricas que possibilitaram a exclusão de eventual etiologia infeciosa, autoimune, hematológica, bem como a existência de patologia tiroideia, tuberculose ou sarcoidose, que pudessem justificar o quadro clínico observado. A radiografia de tórax auxiliou na exclusão de existência de hipotransparência hilar bilateral, que seria sugestiva de síndroma de Lofgren. 
Neste caso clínico, o agente etiológico do eritema nodoso foi a terapêutica contracetiva com desogestrel, tendo-se observado uma evidente melhoria das queixas álgicas e das lesões de eritema após oito dias de suspensão do fármaco e início de prednisolona $5 \mathrm{mg}$ por dia. Um problema encontrado na prática clínica foi a exclusão de infeção do trato urinário como fator etiológico; no entanto, ocorre melhoria clínica evidente quando ainda não foi tratada a ITU, a urocultura isola um Proteus mirabilis resistente à antibioterapia empírica até então instituída (amoxicilina ácido clavulânico) e apenas posteriormente a doente é medicada com uma cefalosporina de acordo com o antibiograma (cefuroxime 500mg de $12 \mathrm{em} 12$ horas).

A toma de desogestrel constitui uma causa rara de eritema nodoso, sabe-se que este se inclui no grupo de efeitos secundários raros (de acordo com o resumo das características do medicamento do fármaco), ocorrendo em menos de uma por cada 1.000 pessoas. ${ }^{8}$ De acordo com o estado da arte, a infeção por Proteus mirabilis já foi previamente mencionada como responsável por quadros de eritema multiforme. ${ }^{9}$

O caso clínico apresentado pretende evidenciar uma causa rara de eritema nodoso, a toma de desogestrel, a importância do diagnóstico para o médico de medicina geral e familiar e de medicina interna e a importância da adequada articulação entre especialidades médicas na gestão do diagnóstico, terapêutica e acompanhamento do doente.

Para o médico de família, assim como para o médico de medicina interna, é importante ter presente as manifestações clínicas da patologia bem como o facto de um fármaco frequentemente utilizado poder ser a causa destas manifestações, isto é, embora as manifestações sejam raras o uso do fármaco é frequente. O médico de família é quem está mais próximo, quem melhor conhece o doente e o segue ao longo do seu ciclo de vida, sendo junto do médico de família que as mulheres jovens têm consulta de planeamento familiar. Um caso clínico semelhante pode apresentar-se numa consulta da especialidade de medicina geral e familiar e cabe ao médico assistente esclarecer o agente etioló- gico. A adequada articulação entre especialidades médicas é o caminho certo no esclarecimento diagnóstico, tratamento e acompanhamento do doente, constituindo este caso clínico um exemplo desta articulação.

\section{REFERÊNCIAS BIBLIOGRÁFICAS}

1. Hebel JL. Erythema nodosum. Medscape [Internet]; 2018 [updated 2020 May 11; cited 2019 Nov 5]. Available from: https://emedicine.medscape.com/article/1081633-overview

2. Chowaniec M, Starba A, Wiland P. Erythema nodosum: review of the literature. Reumatologia. 2016;54(2):79-82.

3. Kroshinsky D. UpToDate [Internet]; 2018 [updated 2021 May; cited 2019 Nov 5].Available from: https://www.uptodate.com/contents/erythema-nodosum

4. Ponte C, Lobo FA. Eritema nodoso: da consulta do médico de família ao diagnóstico [Erythema nodosum]. Rev Port Clin Geral. 2007;23 (5):541-5. Portuguese

5. Bernardes Filho F, Quaresma MV, Vendramini DL, Bonkevitc F, Damiani L, Alves AO, et al. Eritema nodoso pós-infecção de via aérea superior: a propósito de dois casos [Erythema nodosum post-infection of the upper airway: apropos of two cases]. Rev SPDV. 2013;71(3):361-4. Portuguese

6. Hafsi W, Badri T. Erythema nodosum. In: StatPearls [Internet]. Treasure Island: StatPearls Publishing; 2019 [updated 2020 Set 20; cited 2019 Nov 5]. Available from: https://www.ncbi.nlm.nih.gov/books/ NBK470369/

7. Pereira CM. Eritema nodoso: um relato de caso [Erythema nodosum: a case report]. Rev Port Med Geral Fam. 2019;35(5):417-23. Portuguese

8. Sandoz. Desogestrel [Internet]. São Paulo: Sandoz; 2014 [cited 2021 Jun 9]. Available from: https://www.sandoz.com.br/sites/www.sandoz. com.br/files/PF-Desogestrel.pdf

9. ShelleyWB. Bacterial endotoxin (lipopolysaccharide) as a cause of erythema multiforme. JAMA. 1980;243(1):58-60.

\section{CONFLITO DE INTERESSES}

Os autores declaram não possuir quaisquer conflitos de interesse.

\section{FONTES DE FINANCIAMENTO}

Os autores declaram não possuir quaisquer fontes de financiamento.

\section{ENDEREÇO PARA CORRESPONDÊNCIA}

Filipa Sofia Silva

E-mail: filipa.silva@campus.ul.pt

https://orcid.org/0000-0003-3052-6815

Recebido em 27-01-2020

Aceite para publicação em 07-05-2021 


\section{ABSTRACT}

\section{AN EXUBERANT CASE OF ERYTHEMA NODOSUM ASSOCIATED WITH DESOGESTREL}

Introduction: Erythema nodosum is an acute nodular erythematous eruption usually limited to the extensor aspects of the lower legs. The most common causes are streptococcal infections in children and streptococcal infections and sarcoidosis in adults. Case description: Authors present a clinical case of a 33-year-old woman who attends the emergency department with polyarthralgia with one week of evolution, associated with painful erythematous nodules in the lower legs suggestive of erythema nodosum. It is described the whole study and follow-up performed in internal medicine appointment, identifying the etiology, desogestrel, and excluding other possible etiologies.

Comments: The clinical case presented a favorable evolution, with complete resolution of symptoms and skin eruptions, after etiological study and therapeutic approach.

Keywords: Erythema nodosum; Etiology; Contraceptive agents. 